\title{
Glass Gob Modeling and Experimental Validation Using a Drop Test
}

\author{
Adrià Biosca $^{1,2}$, Salvador Borrós ${ }^{1}$, Vicenç Pedret Clemente ${ }^{2}$ and Andrés-Amador García Granada ${ }^{1, a}$ \\ ${ }^{1}$ IQS School of Engineering, 08017 Barcelona, Spain \\ ${ }^{2}$ Ramon Clemente, 08320 El Masnou, Spain
}

\begin{abstract}
Glass blowing to create bottles with specific thickness distribution profiles requires several experimental iterations. Such iterations are expensive and increase the time to market. The use of simulation pretends to decrease the amount of prototypes by doing virtual validation of glass blowing molds. To feed simulations with realistic physical values, a gob drop test has been designed. This test provides valuable experience on the use of the software and validates heat transfer, viscosity and other physical parameters. Gob drop test was chosen for the possibility to record the test with infrared thermal cameras. Gob obtained similar shapes when dropped on a cast iron plate for both central and side sections with longer cooling of about $25^{\circ} \mathrm{C}$. Such technique allowed the user to gain experience on the use of software and obtain valuable physical parameters for future glass blowing optimization.
\end{abstract}

\section{Introduction}

Glass containers have been used during ages. For packaging industry certain qualities are required, and glass has most of them: it is an innocuous and hermetic material that allows long-term preservation, it is a recyclable material and it adds a lot of value to the final product. The individual machine section system (IS) was implemented at the beginning of the 20th century, first as manual machines and later as automatic machines.

Ramon Clemente (RC) is a glass container manufacturing company for the perfume sector. It has about 75 years experience, but like many other glass companies, developing new containers and its mold equipment is still made in the old fashioned way. There is a lot of empirical knowledge on molds design, but at the end most of it is reduced to trial and error. This means that for every new container development, new molds are designed, machined and tested at the production line. The formed containers are analyzed and adjustments are made in the mold and in the process conditions until desired quality is reached.

Numerical simulations of glass forming process are complex because there is a conjugated heat transfer problem, glass viscosity has a very high dependence with temperature, glass domain suffers extreme free surface deformations, etc. Over the last decades, taking advantage of the development and improvement of numerical simulations, the narrow neck pressed blow (NNPB) forming process has been studied to optimize the weight of the containers [1-3].

\footnotetext{
${ }^{\text {a }}$ Corresponding author : andres.garcia@iqs.edu
} 
To speed delivery times, reduce costs and plant downtime, a simulation tool capable to predict container glass distribution in function of blank mold cavities and production process properties it is being developed. The success of this project would help RC to be much more competitive.

\section{Glass forming process description}

Glass forming process begins with the melting of raw materials in the furnace, refining in the working end and conditioning the molten glass in the forehearth. The glass stream flows through the feeder and the orifice ring and it is cut by shear blades forming discrete gobs. Delivery equipment distribute the gobs to the machine sections where container forming occurs.

Many forming steps are needed to manufacture a container with an IS machine, the whole process can be seen in Figure 1: glass starts being a gob loaded inside the blank mold, after two blows in opposite directions the parison shape has been defined; an arm transfers the parison from the blank to the blow mold where glass parison is reheated, then it stretches and is blowed again. Final containers are sent through an annealing lehr, where its internal stresses are released. At the beginning glass behaves as a fluid and ends up being a solid due to the temperature dependence on viscosity.

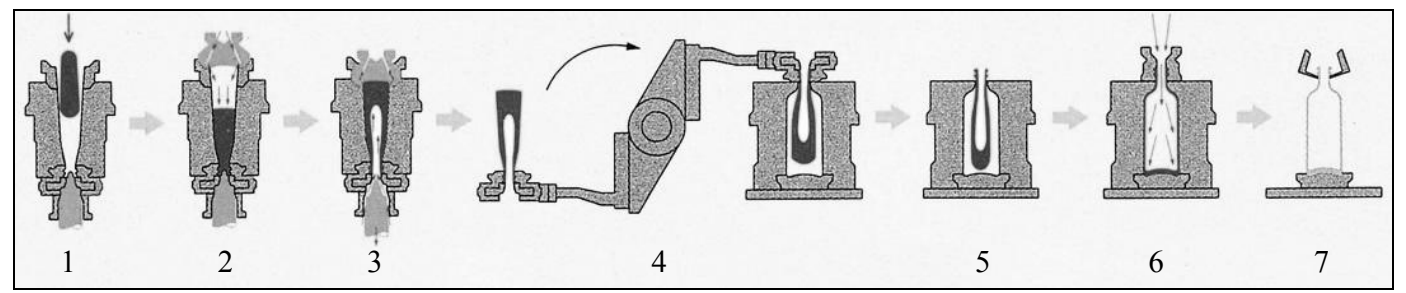

Figure 1. Container forming IS machine stages for blow and blow process. Described stages are: gob loading (1), settle blow (2), counter blow (3), transfer (4), reheat and stretch (5), final blow (6) and take out (7).

\section{Gob drop validation for future blowing simulation}

The development of a simulation tool capable of predicting the final container glass thickness distribution in function of the blank mold cavities and production process conditions is a very complex problem. Moreover, running the simulations requires a lot of sensitive information (i.e. material properties, initial values, boundary conditions, etc.) that needs to be previously obtained from the process itself.

Understanding, interpretation and validation of the results is also complex, as most of the time during the production process, glass remains inside the blank or blow mold. It is hard to predict initial and final glass geometries inside the molds, and define its transition during blowing because the lack of visibility on closed molds. Only the use of thermocouples installed on the mold cavity surface as a contact sensors [4] may show some clues about the flow of glass.

Due to all the modeling difficulties, it seemed interesting to work on a simpler case like studying glass deformation during a gob drop test. This case has been an initial step that has helped to solve many modeling and data acquisition issues. In addition, drop test simulations have been helpful to obtain a better understanding of the model simulations. All the improvements achieved with the drop test have been also used to improve the ongoing simulations of the blow and blow model.

The objective of this paper is to correlate numerical and experimental results of a drop test, and to apply the knowledge learned to the blow and blow forming simulations.

\subsection{Gob drop numerical simulation}

A 2D axisymmetric model has been built using ANSYS Polyflow. The simulation describes the impact of the free falling glass gob against a cast iron plate. Glass gob geometry has been simplified 
as a cylinder with rounded edges. Glass volume is described by gob length and diameter, and it is in concordance with the container weight. Gob dimensions were obtained from experimental measurements.

The numerical model contains two domains:

- The glass domain is defined as a generalized Newtonian non-isothermal material. Material data is set, including temperature dependence of viscosity by the Vogel-Fulcher-Tammann viscosity equation [5]. Density, thermal conductivity and heat capacity per unit mass are also specified.

Gob velocity at impact has been obtained from experimental measurements.

Experimental measurements have been performed to determine glass temperature in the gob skin. During loading gob heat is released mainly by contact with the delivery equipment, temperature distributions in glass gobs at loading stage have been a subject of study in the past [6]. A temperature gradient has been defined from the skin to inside the gob. Such temperature distributions were obtained from transient cooling simulations during the time that the gob travels from the exterior of the feeder to the blank mold, through the delivery equipment.

A thermal contact conductance is defined between glass and mold at its contact interference, convection heat loss is defined too.

- The plate domain is also defined as non-isothermal. In order to describe a similar thermal contact between glass and mold than in the loading, material properties from blank mold cast iron were used to set the plate. Density, thermal conductivity and heat capacity per unit mass are also specified.

Due to the extreme high deformations of the glass domain, an adaptive remeshing has been configured, the quality of the mesh elements is checked after each time step. A finer mesh has also been defined near the interference of the glass with the mold to correctly define the thermal gradient. Volume conservation is verified so as not to modify the initial volume of the glass domain with all the mesh modifications.

\subsection{Gob drop experimental testing}

A plate has been machined using the same cast iron material as the blank mold and with the same surface finish as in the mold cavities. This plate has been fixed on the funnel. The funnel arm is blocked in loading position and all other individual section (IS) movements are also blocked. Discrete gobs are loaded through the delivery equipment to the plate to perform the drop test.

A FLIR A325sc infrared thermal camera has been used to measure the temperatures in a range from 300 to $2,000^{\circ} \mathrm{C}$ and also the glass deformation along the impact was recorded.

Tests were performed under different conditions described in the results and discussion section.

\section{Results and discussion}

Tests were performed on a six section IS machine with a single gob (SG) cooled distributor. Several gob drop tests and simulations have been performed under different working conditions.

During the gob drops different stages can be defined describing the glass gob mechanical behavior:

1. The drop test starts with the gob at a certain height and zero velocity.

2. The gob accelerates during free fall until the impact velocity is reached.

3. The gob strikes the surface of the cast iron plate.

4. During the impact the gob undergoes extreme deformation and flows over the plate surface, the initial shape of a cylinder ends up being a kind of amorphous medallion.

5. The glass stops flowing due to heat loss at mold contact interface and on the skin.

\subsection{Gob drop test conditions: delivery equipment}

Height between the shear blades and the top of the blank mold is about $2.45 \mathrm{~m}$, this is the free fall gob height. In a free fall scenario, the total fall time would be $0.707 \mathrm{~s}$ and impact velocity would be 
$6.93 \mathrm{~m} / \mathrm{s}$. But at the IS machine, between the shear and the funnel and the blank mold there is the delivery equipment, which is formed by three different parts: scoop, trough and deflector.

The orifice ring and the shear are situated in the middle of the IS machine and they describe a symmetry axis when the IS machine is viewed from the front view, so on the left and right side there are three IS which are symmetric. IS1 delivery equipment is symmetric with IS6 (side sections), IS2 with IS5 and IS3 with IS4 (central sections).

There is one scoop for all six sections. There are six troughs which are symmetrical; central IS troughs are shorter than side ones. The slope of all the troughs is almost the same. There are six deflectors which also are symmetrical; all the deflectors have a similar curvature, but at the end a straight section is added for the central IS, so central IS troughs are longer than side ones (see Table 1).

Table 1. Delivery equipment (DE) length measurements (m) in function of IS machine.

\begin{tabular}{|c|c|c|c|}
\hline & IS1 (side) & IS2 & IS3 (central) \\
\hline Scoop & 0.62 & 0.62 & 0.62 \\
\hline Trough & 1.22 & 0.85 & 0.61 \\
\hline Trough length increase & $+100.0 \%$ & $+39.3 \%$ & $0 \%$ (reference) \\
\hline Deflector & 0.56 & 0.63 & 0.72 \\
\hline Deflector length increase & $-22.2 \%$ & $-12.5 \%$ & $0 \%$ (reference) \\
\hline Total DE length & 2.40 & 2.10 & 1.95 \\
\hline Total DE length increase & $+23.1 \%$ & $+7.7 \%$ & $0 \%$ (reference) \\
\hline
\end{tabular}

The fact that there are six IS machines and the orifice ring and the gob distributor with the scoop are between IS3 and IS4, defines that: IS3 and IS4 (central sections) are closer and gobs travel at close range; On the other hand, S1 and IS6 (side sections) are further away and gobs travel farther. This delivery equipment differences between sections have consequences for the glass properties, comparing IS1 with IS3:

- The gob displacement distance from orifice ring to the blank mold is longer, and the gob needs more time to be delivered.

- Longer distance and longer delivery equipment mean longer contact time and more friction between glass and the delivery equipment.

- Friction with the delivery equipment affects the shape of the gob, during the falling the gob is lengthened.

Therefore, in the end, there is more heat loss on the gob surface due convection and contact conductance with the delivery equipment; consequences of that heat loss can also be seen on [6]. The gob impact velocity will be lower. There is no appreciable impact on gob elongation.

Gob drop tests and its simulations have been performed under IS1 and IS3 working conditions.

\subsection{Gob drop experimental results}

Gob drop tests were performed from the central and side sections of the IS machine.

The temperature of the glass gobs has been measured at the end of the three IS deflectors. Figure 2 shows the temperature values of 14 consecutive gobs loaded at each individual section. It can be appreciated how IS1 temperature values are lower than IS2 and IS3. Deviation from all three series is under $\pm 5^{\circ} \mathrm{C}$ when compared to the mean values. Side section gobs showed higher cooling with lower 
glass temperatures of around $25^{\circ} \mathrm{C}$ when compared to central section gobs with a good statistical repeatability.

Total fall time and impact velocity are also consistent: side section fall times are longer than central section ones and impact velocities show slower values when compared to central section velocities. However, there is no appreciable impact on gob elongation.

Figure 3 shows four drop tested gob samples, IS1 on the left and IS3 on the right. Although gob velocity and surface temperature before impact were lower, its final dimensions are almost the same. There is no appreciable difference on the final glass drop tested samples when side and central sections are compared.

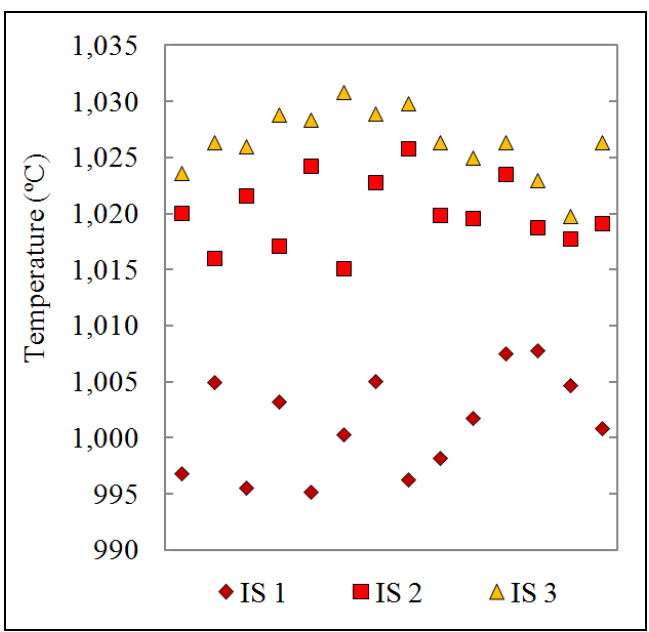

Figure 2. Gob temperature measurements.

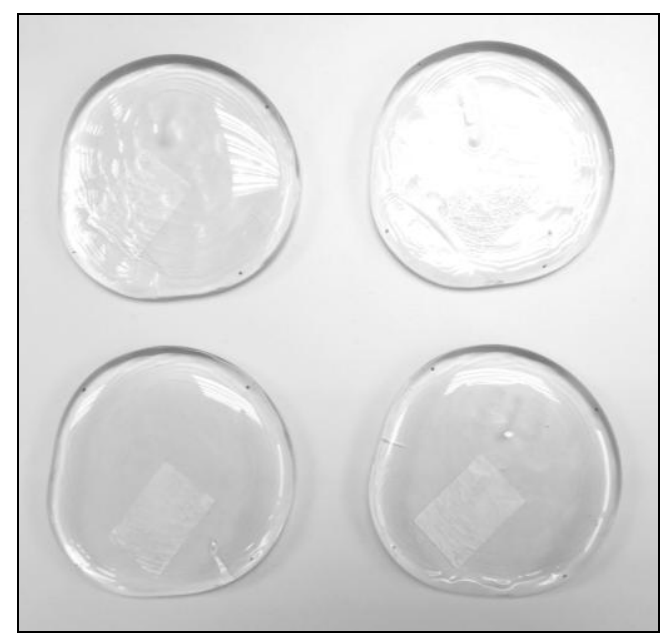

Figure 3. Drop tested gob samples.

Deflectors can be adjusted to guide the gob's path and perform a normal impact against the surface of the horizontal plate. Despite that, observing the amorphous deformed gob samples no circular symmetry can be found. During deformation, the gob is slightly offset from the vertical axis.

\subsection{Gob drop simulation results}

Gob drop test simulations have been performed under different working conditions. The glass temperature distributions and the impact velocities have been set to reproduce the drop test conditions at central and side sections of the IS machine.

Figure 4 shows representative deformation results from different simulation time steps. Each time step has local scale temperature contours in order to appreciate big temperature gradients in the final step (E), but also the small temperature gradients of (A).

(A) shows the temperature gradient from the skin to inside the gob before impact. (B) and (C) are intermediate simulation steps that describe gob deformation due to impact energy. At (D) most of the kinetic energy has been used to deform the initial gob shape.

But glass is at high temperature and its low viscosity allows it to flow over the plate surface, reason why the deformation of the glass continues at a slower rate. The contact conductance defined in the interface between the glass and the plate removes heat from the glass. The viscosity of the glass increases and after a long period of time, glass stops flowing (E). Without a non-isothermal model, the glass would have flowed indefinitely, obtaining very different results.

The shape of the gob and its skin temperature has been recorded with the IR thermal camera. Experimental and numerical results can be compared in Figures 4 and 5. 


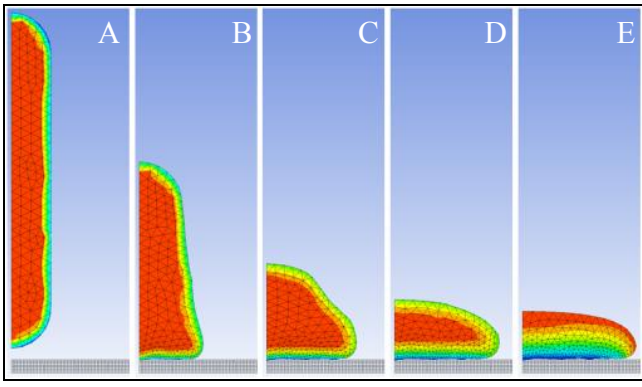

Figure 4. Representative simulation steps.

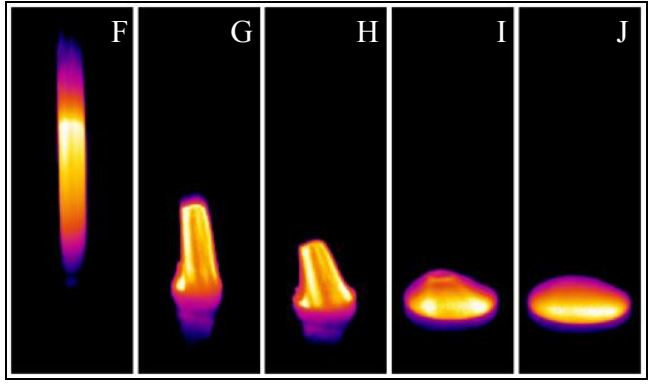

Figure 5. Representative IR captures.

As it can be seen, there is a good correlation between numerical and experimental results. Both results show a good agreement but in the future more drop tests will be performed in order to obtain stronger correlations.

There are no relevant differences in the results of the glass dropped geometries when comparing the simulation results at the side and central sections.

Simulations have been done with a $2 \mathrm{D}$ axisymmetric model, so the model cannot describe symmetry axis deviations of the experimental results.

\section{Conclusions}

An experimental gob drop test case has been designed and numerically modeled. This test provides valuable experience on the use of the software and validates heat transfer, viscosity and other physical parameters.

The temperature distribution in the glass domain defines its viscosity and flow. As seen in the numerical results, a non-isothermal model is required to describe the viscosity highly dependence on temperature to reproduce the glass flow of the experimental results. Apparently, an isothermal model will also not be suitable for describing the glass flow in blow and blow glass container forming simulations.

Gob loading conditions at the central and side IS machine sections have been studied and initial assumptions have been verified. Side section gobs showed higher cooling with lower glass temperatures of around $25{ }^{\circ} \mathrm{C}$ when compared to central section gobs with a good statistical repeatability. The total fall time and impact velocity are also consistent: side section fall times are longer and impact velocities are slower than central section ones.

Initial gob conditions are different depending on the section, however experimental and numerical results do not show relevant differences.

\section{References}

1. J.M.A. Cesar de Sa, Eng. Computation, 3 [4], 266-275 (1986).

2. M. Hyre, J. Am. Ceram. Soc., 85 [5], 1047-1056 (2002).

3. J.A.W.M. Groot, C.G. Giannopapa, R.M.M. Mattheij, J. Press. Vess.-T. Asme, 136, 1-9 (2014).

4. D.M. Shetterly, N.T. Huff, J. Non-Cryst. Solids, 38 39, 867-872 (1980).

5. G.S. Fulcher, J. Am. Ceram. Soc., 8 [6], 339-355 (1925).

6. H. Müller-Simon, G. Bergmann, K. Kessler, 70th Conference on Glass Problems, 151-158 (2010). 